\title{
Androgen receptor and heat shock protein 27 co-regulate the malignant potential of molecular apocrine breast cancer
}

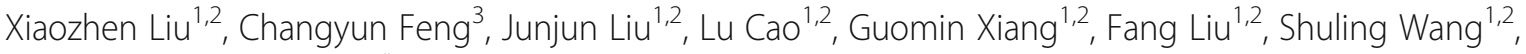
Jiao Jiao ${ }^{1,2}$ and Yun Niu ${ }^{1,2^{*}}$ (D)

\begin{abstract}
Background: The most striking feature of molecular apocrine breast cancer (MABC) is the expression of androgen receptor (AR). We report here the mechanism of the $A R$ in regulating the behavior of MABC.

Methods: The MABC cell line, MDA-MB-453, and the nonMABC cell line, MCF7, were used in this study. The effect of dihydrotestosterone (DHT) and heat shock protein 27 (HSP27) on cell proliferation was quantified using the cell counter kit-8 (CCK8) and clonogenic assays in vitro and by a xenograft tumor model in vivo. The expression of the AR and HSP27 was analyzed using western blot, QPCR, and immunofluorescence assays. Complexes of the AR and HSP27 were detected by co-immunoprecipitation (Co-IP).

Results: In MDA-MB-453 cells, DHT promoted cell proliferation and stimulated AR and HSP27 translocation from the cytoplasm to the nucleus, whereas, it inhibited MCF7 cell growth, and only the AR translocated into the nucleus. HSP27 knock-down decreased the proliferative ability of MDA-MB-453 cells, which could be rescued by DHT, while HSP27 and DHT had synergistic effects on MCF7 cells. HSP27 phosphorylation was a prerequisite for AR translocation into the nucleus, especially phosphorylation on serine 82. In addition, DHT stimulated the tumorigenic and metastatic capacities of MDA-MB-453 cells, while HSP27 knock-down decreased the rate of tumor formation and induced apoptosis in cells.
\end{abstract}

Conclusions: The results suggest that HSP27 assists the AR in regulating the malignant behavior of MABC, and these findings might be helpful in the treatment of MABC.

Keywords: Androgen receptor, Heat shock protein 27, Molecular apocrine, Phosphorylation

\section{Background}

As a member of the nuclear receptor family, the androgen receptor (AR) plays an important role in breast cancer, and has been identified as a biomarker for a specific molecular subtype of breast cancer. The AR gene expression profile can be used for further classifying receptor-negative tumors as molecular apocrine breast

\footnotetext{
* Correspondence: yunniu2000@126.com

'Department of Breast Cancer Pathology and Research Laboratory, Tianjin Medical University Cancer Institute and Hospital, National Clinical Research Center of Cancer, Key Laboratory of Cancer Prevention and Therapy, Tianjin, Tianjin's Clinical Research Center for Cancer, Tianjin 300060, China

${ }^{2}$ Key Laboratory of Breast Cancer Prevention and Therapy, Tianjin Medical University, Ministry of Education, West Huanhu Road, Ti Yuan Bei, Hexi District, Tianjin 300060, China

Full list of author information is available at the end of the article
}

cancer (MABC) [1]. MDA-MB-453 breast cancer cells have been classified as molecular apocrine by gene profiling studies [2]. Our previous study has stated that patients with MABC develop distant metastases earlier and have poor prognosis [3]. As MABC is characterized by increased androgen signaling, the malignant potential of MABC may partly be because of the AR. A study has shown that when the AR in MDA-MB-453 breast cancer cells is knocked down, the cell colony formation rate is significantly decreased, which verifies the fact that the AR regulates the biological behavior of MABC [4]. Both ligand binding and translocation from the cytoplasm to the nucleus play important roles in the function of the AR [5]. However, the specific mechanism of cytoplasmic

(C) The Author(s). 2018 Open Access This article is distributed under the terms of the Creative Commons Attribution 4.0 International License (http://creativecommons.org/licenses/by/4.0/), which permits unrestricted use, distribution, and reproduction in any medium, provided you give appropriate credit to the original author(s) and the source, provide a link to the Creative Commons license, and indicate if changes were made. The Creative Commons Public Domain Dedication waiver (http://creativecommons.org/publicdomain/zero/1.0/) applies to the data made available in this article, unless otherwise stated. 
and nuclear translocation of the AR has not been clarified in MABC.

In the absence of ligand, the AR remains in a nonactive state, which forms a protein complex with heat shock proteins (HSPs) and other co-chaperones in prostate cancer, while the AR becomes active when ligand binds, and it translocates to the nucleus as a transcriptional regulator [6]. Based on this result, HSPs play crucial roles in the process of AR activation. As a member of the molecular chaperones, HSP27 forms a chaperoning oligomer which can regulate multiple cellular survival and signaling pathways [6]. However, whether HSP27 combines with the AR during its translocation from the cytoplasm to the nucleus in MABC cells remains unclear. Furthermore, in the process of AR translocation to the nucleus, the rapid phosphorylation of HSP27 is regulated by ligand binding [6, 7]. There are three serine residues, serine 15,78 , and 82 , reported as the sites of human HSP27 phosphorylation $[8,9]$. However, which one of these residues plays an indispensable role for AR cytoplasmic and nuclear translocation still remains unknown.

To detect the specific mechanism of AR cytoplasmic and nuclear translocation in MABC cells, androgen and siRNAs specific for HSP27 were used to analyze the location of the AR and HSP27 in vitro, and their effects on the tumorigenic capacity of MABC cells in vivo. The results of this study could determine the mechanism of the AR in regulating the malignant potential of MABC. Additionally, it aims to explore potential therapeutic targets for patients with MABC.

\section{Methods}

\section{Cell lines and culture}

Because the MDA-MB-453 cell line is classified as a model of MABC [2], and MCF7 cells as nonMABC cell line [10], we obtained them from American Type Culture Collection (ATCC, USA) for this study. The MDA-MB-453 cells were cultured in L15 medium (Gibco, USA), containing 10\% fetal bovine serum (FBS, Gibco, USA) and $1 \%$ penicillin/streptomycin (Life Technologies, USA). MCF7 cells were cultured in DMEM medium (Gibco, USA) which contained 10\% FBS and 1\% penicillin/streptomycin. Both cell lines were incubated at $37{ }^{\circ} \mathrm{C}$ in $5 \% \mathrm{CO}_{2}$.

\section{Plasmids and transfection}

The HSP27 siRNAs and control plasmids were constructed by Genechem (China). Three target sequences for the HSP27 siRNAs were studied, which included siRNA\#4892-1: 5' -CTGTGAGGACTGTGGATAA-3', si RNA\#4893-1: 5' -CCCAGCAAATCCCTCTCTA-3' and siRNA\#4894-2: 5' -GGCAAGTTCCAGGCATTT-3'.
The deletion of HSP27 phosphorylation sites (Ser15, Ser78 and Ser82; CS-I0586-Lv201-01, CS-I0586-Lv20102, and CS-I0586-Lv201-03) were carried out by GeneCopoeia (China, Additional file 1). The plasmids were amplified in $E$. coli, and then extracted by using the Endotoxin-free Plasmid Size Kit (TIANGEN, China).

Cell transfections were performed as follows: firstly, cells were seeded in 6-well plates at a density of $1.0 \times$ $10^{4}$ cells per well overnight. Subsequently, $2 \mu \mathrm{g}$ of the constructed plasmids were added to MEM (Gibco, USA) , respectively, and incubated for $5 \mathrm{~min}$ at $37^{\circ} \mathrm{C}$. Further, the FuGENE Transfection Reagent (Promega, USA) was added and mixed. After incubating for $15 \mathrm{~min}$, the complex solution was added to the cells, and replaced with complete medium $8 \mathrm{~h}$ later. The reactions were incubated for $48 \mathrm{~h}$.

\section{Quantitative real-time PCR (qPCR)}

The RNAs used in this study were extracted using Trizol reagent (Takara, Japan). Reverse transcription was carried out using the SuperScript RT kit (Takara, Japan). qPCRs were performed according to the manufacturer's protocol using the SYBR Green PCR kit (Toyobo, Japan). The transcript level of GAPDH was adopted as an internal control, and the primers used were as follows: GAPDH: 5'-GGAAGGTGAAGGTCGGAGTC-3' and 5'-GTCTTCTGGGTGGCAGTGAT-3'; AR: 5'-GGAA TTCCTGTGCATGAAA- $3^{\prime}$ and $5^{\prime}$-CGAAGTTCAT CAAAGAATT-3'; HSP27: 5' - GCGTGTCCCTGG ATGTCAAC-3' and ${ }^{\prime}$ '-TGTATTTCCGCGTGAAG CAC-3'. Each sample was assayed in triplicate.

\section{Western blot analysis}

Total proteins were extracted with RIPA buffer (Thermo Scientific, USA) and $1 \mathrm{mM}$ PMSF. Cytoplasmic and nuclear subcellular fractionation was performed according to the manufacturer's instructions using the Nuclear and Cytoplasmic Isolation Kit (KeyGEN, China). All proteins were separated on $10 \%$ SDS-PAGE (Invitrogen, USA) gels, transferred onto PVDF membranes (Millipore, USA), and then blocked using 5\% skim milk. The proteins were detected by incubating the following primary antibodies: anti-AR (AR441; Abcam, USA), anti-estrogen receptor (ER; D8H8; CST, USA), anti-progesterone receptor (PR; 6A1; CST, USA), anti-HSP27 (G3.1, Abcam, USA), anti-HSP27 (phospho S15) (EP2293Y, Abcam, USA), anti-HSP27 (phospho S78) (Y175, Abcam, USA), anti-HSP27 (phospho S82) (EPR7278, Abcam, USA), anti- $\beta$-actin (8H10D10, CST, USA), and anti-Histone H3 (D18C8, CST, USA) overnight; and incubated with horseradish peroxidase-labeled anti-rabbit or anti-mouse IgG, followed by detection using the ECL detection kit (Solarbio, China). Each sample was analyzed in triplicate. 
Co-immunoprecipitation(Co-IP) and western blot

Co-IP of the AR and HSP27 was carried out according to the manufacturer's protocol using the Pierce Co-IP kit (Thermo Scientific, USA). Briefly, AR or HSP27 antibody $(10 \mu \mathrm{L})$ was first incubated with the AminoLink Plus coupling resin. The antibody-coupled resin was incubated with protein lysates overnight. Subsequently, the resin was washed, followed by elution of the protein complexes, which bound to the AR or HSP27 antibody. Subsequently, the samples were detected by western blot using the HSP27 or AR antibody as described previously. Each sample was assayed in triplicate.

\section{Cell counter kit-8 (CCK8) cell proliferation and clonogenic assays}

Cells (DHT treatment or HSP27 knock-down) were suspended and seeded in 96-well plates at 5000 cells per well and incubated for $24 \mathrm{~h}$. Further, $10 \mu \mathrm{L}$ of CCK8 (KeyGEN, China) solution was added into each well on day 1, 2, 3, 4 and 5. After 1-4 h, the absorbance of each well was measured at $450 \mathrm{~nm}$ using a microplate reader. Clonogenic assays were carried out using 6-well plates with 1000 cells per well. After 15 days, cells were collected and stained with crystal violet, and then the number of cell colonies was counted. Each sample was assayed in triplicate.

\section{Immunofluorescence (IF) assay}

Cells were seeded in 24-well plates. After $24 \mathrm{~h}$, cells were fixed with $4 \%$ paraformaldehyde, permeabilized with $0.2 \%$ Triton X-100, blocked with bovine serum albumin, incubated with the primary antibodies and fluorescein-labeled secondary antibody, and then DAPI (Thermo Scientific, USA) was used to stain the nucleus. Images were visualized and analyzed using a fluorescence microscope. Each sample was analyzed in triplicate.

\section{In vivo experiments}

Eighteen female BALB/c-nude mice (4-6 weeks old, 18-20 g) were purchased and randomly divided into three groups: MDA-MB-453 cells with DHT treatment, HSP27 knock-down, and the control group. Cells $\left(2 \times 10^{6}\right)$ were inoculated subcutaneously in the groin. Care and procedures of the mice were provided by the Institution of Animal Use and Care Committee of Tianjin Medical University Cancer Institute and Hospital. All mice were sacrificed until 55 days. Tumor volumes were calculated as previously reported [11].

All the tumors, livers, and lungs were paraffinembedded and stained with hematoxylin-eosin (HE). HSP27 and Ki67 expression of mouse tumors were analyzed by immunohistochemistry as previously reported
[3]. In order to detect apoptotic cells, the terminal deoxynucleotidyl transferase dUTP nick end labeling (TUNEL) assay was carried out according to the manufacturer's instructions, and the kit was obtained from KeyGEN (China).

\section{Statistical analysis}

Statistical analyses were performed using SPSS 19.0 software. The data were recorded as means \pm standard deviation from at least three independent experiments, and analyzed by one-way ANOVAs and T-tests. $P<0.05$ was considered as statistically significant.

\section{Results}

Determination of cells and DHT working conditions

Two breast cancer cell lines were used in this study, MDA-MB-453 and MCF7, which are representative of $\mathrm{MABC}$ and nonMABC, respectively. Cells were tested by western blot to determine the expression levels of ER, $\mathrm{PR}$, and AR. MDA-MB-453 cells demonstrated no detectable levels of ER or PR, but high levels of AR, while all of these proteins could be detected in MCF7 cells. However, the level of AR protein was 1.73-fold higher in MDA-MB-453 than in MCF7 cells (Fig. 1a).

To determine whether dihydrotestosterone (DHT) could affect the proliferation of breast cancer cells, MCF7 cells were cultured in 96-well plates in medium containing $10 \%$ dextran-coated charcoal-treated FBS (DCC-FBS) incubated with or without different concentrations of DHT $\left(10^{-6}, 10^{-7}\right.$, and $\left.10^{-8} \mathrm{M}\right)$ for 24,48 , or $72 \mathrm{~h}$. CCK8 assays showed that the effects of DHT were dose and time dependent, as the growth inhibitory effects could work best at a concentration of $10^{-8} \mathrm{M}$ and simultaneously for $48 \mathrm{~h}$ (Fig. 1b). Therefore, a $10^{-8} \mathrm{M}$ concentration for $48 \mathrm{~h}$ was considered as the optimal condition for DHT in the following studies.

\section{Effects of androgen on MABC cell proliferation}

The effect of DHT on MDA-MB-453 cell proliferation when exposed to DHT for 1-5 days was examined with the CCK 8 assay, and the MCF7 cell line was used as a control. A significant anti-proliferative effect by DHT on MCF7 cells could be observed at the fourth day. However, a significant promotion of cell proliferation in MDA-MB-453 cells by DHT treatment was detected at the third day (Fig. 1c). Clonogenic assays also showed that the number of colonies formed by MDA-MB-453 cells treated with DHT increased dramatically compared to the control cells ( $221 \%$ vs. $100 \%$, respectively), while it decreased in MCF7 cells with DHT treatment compared to the control cells (56\% vs. $100 \%$, respectively; Fig. 1d). Therefore, proliferation of MDA-MB-453 and MCF7 cells showed opposite responses to DHT treatment. 

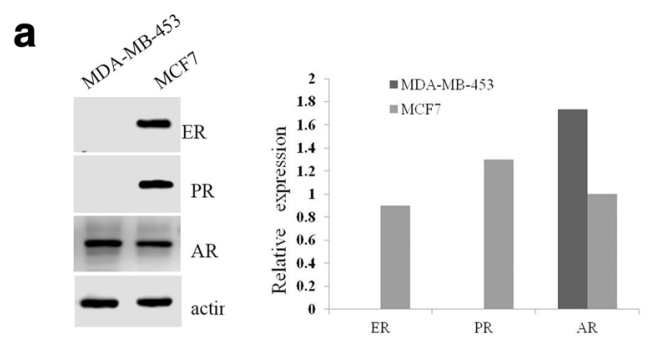

C
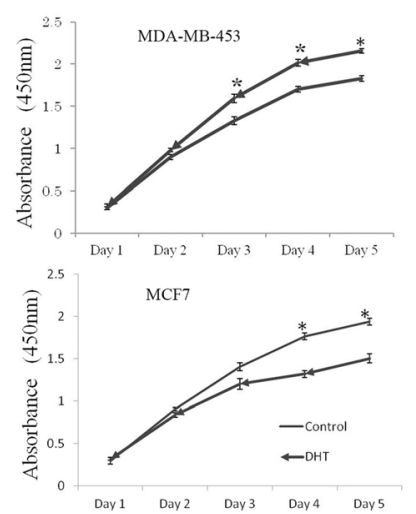

b

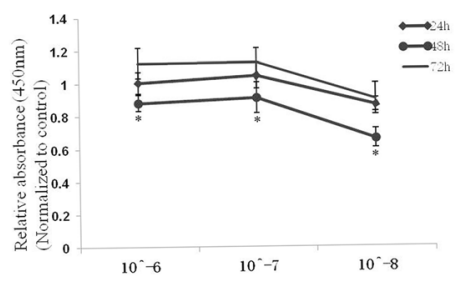

d

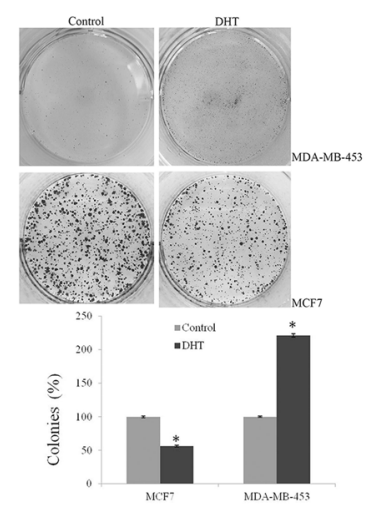

Fig. 1 DHT affected the proliferation ability of breast cancer cells. Western blot analyzed the expression of ER, PR, and AR in MDA-MB-453 and MCF7 cells, and quantified by the bar graph (a). CCK8 assay determined the working conditions of DHT (b). The proliferation ability of MDA-MB-453 and MCF7 cells treated with or without DHT was measured by CCK8 (c) and clonogenic (d) assays. ${ }^{*} P<0.05$

\section{Effects of androgen on AR and HSP27 expression and localization}

To determine whether androgen could affect the expression of the AR and HSP27, western blot was carried out, and the results showed a significant increase in the levels of AR protein in both MDA-MB-453 and MCF7 cells treated with DHT. However, DHT had no effect on the expression of HSP27. As HSP27 is phosphorylated on three serine residues (Ser15, Ser78, and Ser82), the phosphorylated forms of HSP27 after DHT treatment were analyzed. Interestingly, the expression of Ser82 significantly increased, while the others remained unchanged (Fig. 2a). The changes in $A R$ and HSP27 transcript levels in these two cell lines treated with or without DHT were consistent with the changes in protein level. However, the mRNA expression of $A R$ was significantly increased while HSP27expression remained steady-state (Fig. 2b).

Furthermore, the protein levels of the AR and HSP27 in the cytoplasm and nucleus after subcellular fractionation were analyzed. In MDA-MB-453 cells, the levels of both the AR and HSP27 were significantly increased in the nucleus and decreased in the cytoplasm by DHT treatment, while only the level of the AR increased in the nucleus and decreased in the cytoplasm in MCF7 cells (Fig. 2c). In addition, IF assays illustrated that both the AR and HSP27 were localized in the nucleus of MDA-MB-453 cells with DHT treatment compared to the cytoplasm in control cells. However, only the AR was localized in the nucleus of MCF7 cells after DHT treatment (Fig. 2d).

\section{Mechanisms of DHT-induced AR and HSP27 cytoplasmic/ nuclear translocation}

To further confirm the mechanisms of DHT-induced AR and HSP27 translocation from the cytoplasm to the nucleus, the interaction between HSP27 and AR was analyzed by Co-IP methods. Firstly, total proteins were extracted from MDA-MB-453 and MCF7 cells treated with DHT. The results indicated that the AR could be detected in HSP27 immunoprecipitated complexes, and vice versa (Fig. 3a). Subsequently, a cytoplasmic and nuclear subcellular fractionation was performed. Analysis showed that only after DHT treatment, the AR-HSP27 complex could be detected in both the cytoplasm and nucleus of MDA-MB-453 cells (Fig. 3b), and in the cytoplasm of MCF7 cells (Fig. 3c).

\section{Effects of HSP27 on MABC cell proliferation}

Cells transfected with siRNAs specific for HSP27 were examined to explore the effect of HSP27 on AR expression. qPCR and western blot assays determined that HSP27 siRNA\#4893-1 could clearly decrease the expression of HSP27 compared with the others (Fig. 4a and b). CCK8 assays showed that HSP27 knock-down decreased the proliferative ability of MDA-MB-453 cells compared to DHT treated 

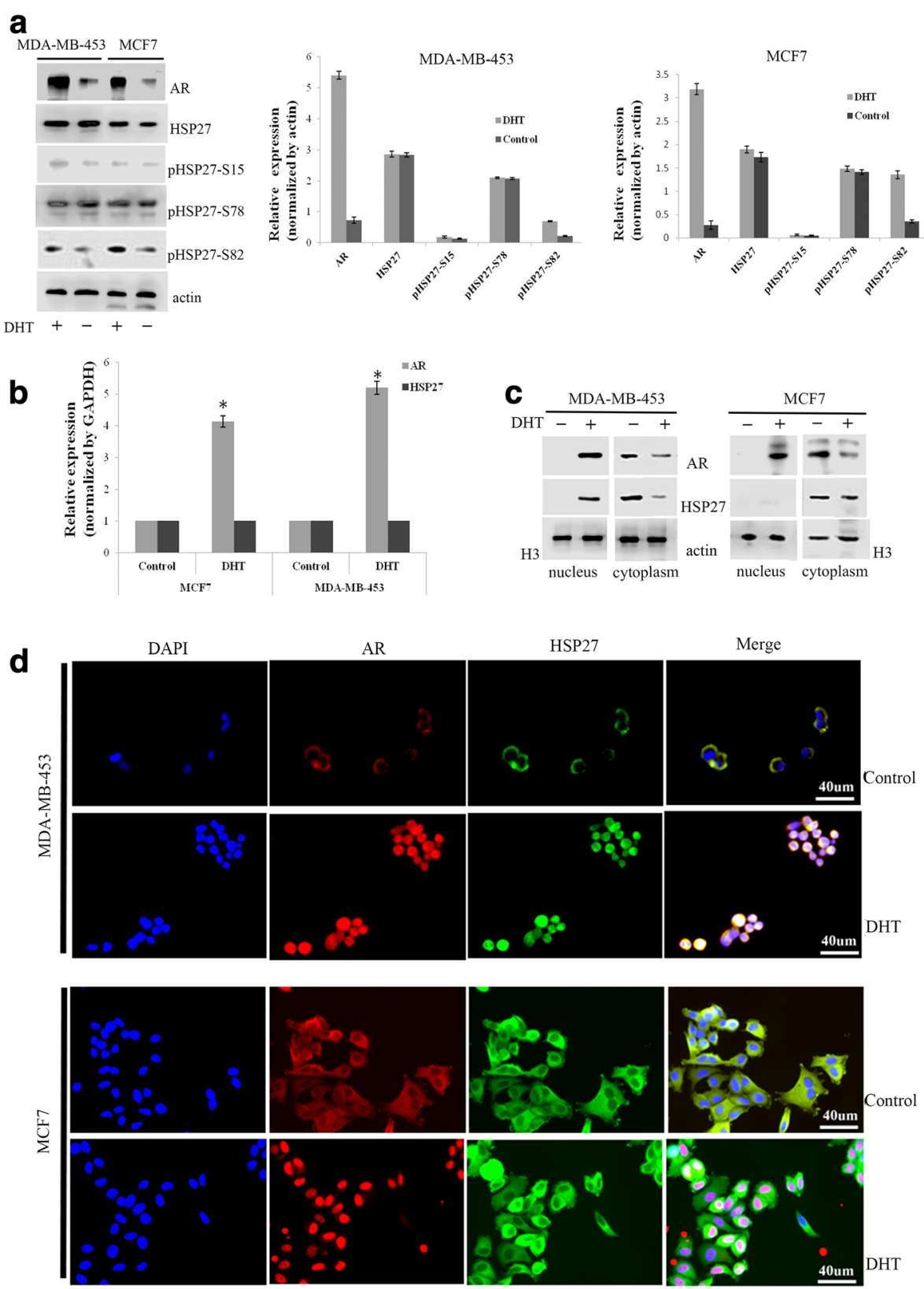

Fig. 2 DHT affected the expression and location of AR and HSP27. The effect of DHT on the expression of AR, HSP27, and phosphorylated forms of HSP27 at serine 15, 78 and 82 was analyzed by western blot (a). The effect of DHT on the expression of AR and HSP27 mRNAs was measured by qPCR (b). The expression and location of AR and HSP27 in the cytoplasm and nucleus after MDA-MB-453 and MCF7 cells treated with or without DHT were analyzed by western blot $(\mathbf{c})$ and immunofluorescence $(\mathbf{d})$ assays. ${ }^{*} P<0.05$

cells. This effect could partly be prevented by DHT treatment. The proliferative ability of MCF7 cells was also decreased by HSP27 knock-down, which was similar to DHT treatment, and was more obvious when cells were treated with both HSP27 siRNAs and DHT (Fig. 4c). Clonogenic assays indicated that for MDA-MB-453 cells, the number of colonies in the HSP27 knock-down group showed a significant decrease compared to the DHT- treated group (27\% vs. $100 \%$, respectively). When cells knocked-down for HSP27 were treated with DHT again, the number of colonies increased (75\%). For MCF7 cells, HSP27 knock-down decreased the number of colonies, almost to the same extent as with DHT (90\% vs. $100 \%$, respectively), and the number was much lower when cells were treated with both HSP27 siRNAs and DHT (62\%, Fig. 4d). 


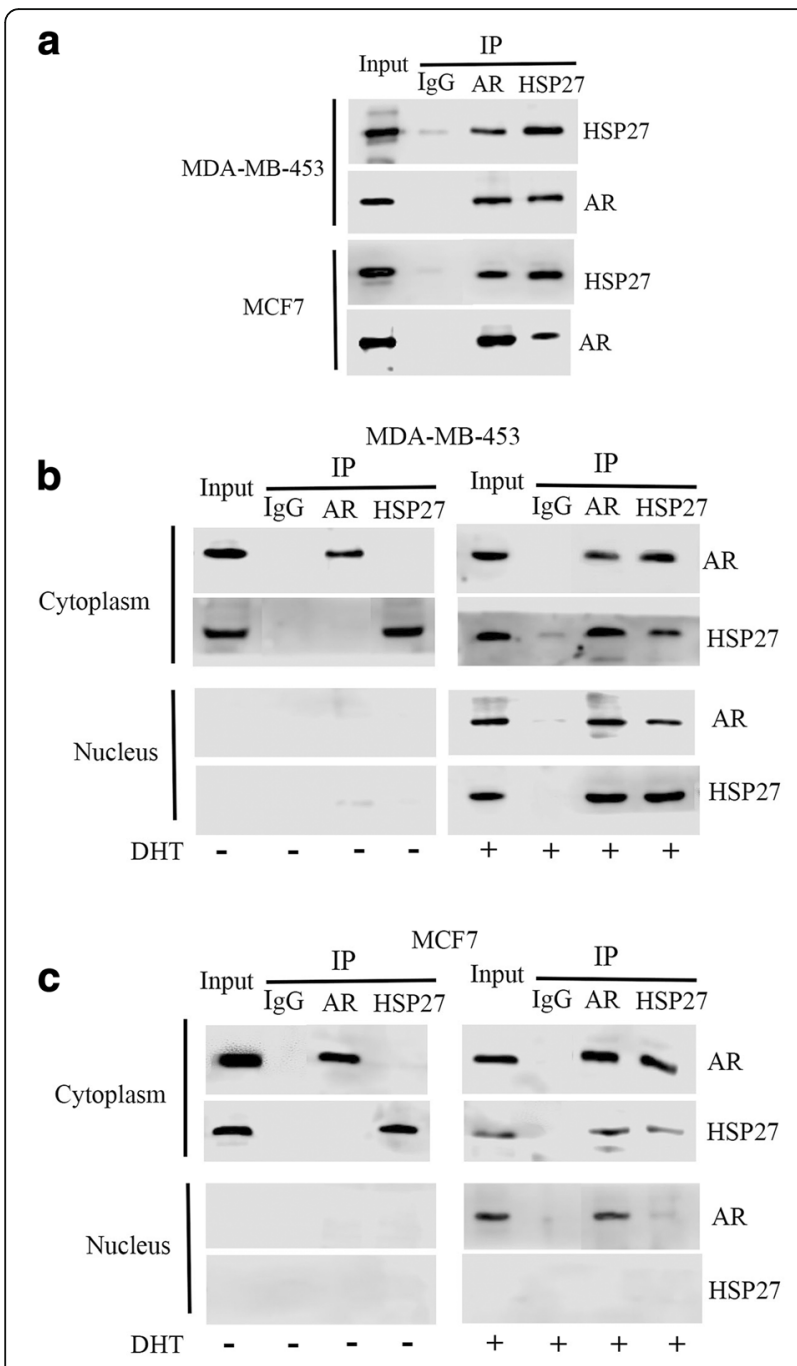

Fig. 3 DHT induced the formation of AR-HSP27 complex. Co-IP for AR and HSP27 in the total proteins extracted from MDA-MB-453 and MCF7 cells treated with or without DHT (a). Co-IP for AR and HSP27 in the proteins extracted from the cytoplasm and the nucleus of MDA-MB-453 (b) and MCF7 (c) cells treated with or without DHT

\section{Effects of HSP27 on AR expression and localization}

The protein level of the AR in HSP27 knock-down cells was detected by western blot, and the results showed that the down-regulation of HSP27 decreased the AR protein level in these cells (Fig. 4e). However, HSP27 knock-down had no influence on the mRNA levels of $A R$ (Fig. 4f). In addition, HSP27 knock-down decreased the level of AR translocation into the nucleus in both MDA-MB-453 and MCF7 cells (Fig. 4g and h).

\section{The critical sites of HSP27 phosphorylation for AR cytoplasmic/nuclear translocation}

Cells transfected with HSP27 serine 15 deleted, HSP27 serine 78 deleted, and HSP27 serine 82 deleted constructs, respectively, were examined to confirm which site would play a critical role in AR cytoplasmic and nuclear translocation. Western blot was performed to verify the effects of the deletions (Fig. 5a). Cytoplasmic and nuclear subcellular fractionations from cells transfected with or without the specific plasmids and treated with DHT were analyzed. Compared to the control group, the level of the AR decreased in the nucleus and increased in the cytoplasm in cells transfected with HSP27 serine 82 deleted, while no significant differences were found in cells transfected with HSP27 serine 15 deleted or HSP27 serine 78 deleted and the control group (Fig. 5b). IF assays further verified that after DHT treatment, both the AR and HSP27 were localized to the cytoplasm of MDA-MB-453 and MCF7 cells transfected with HSP27 serine 82 deleted, compared to the nucleus of cells in the other three groups (Fig. 5c).

\section{Effects of androgen and HSP27 on the tumorigenic capacity of MABC cells}

MDA-MB-453 cells $\left(2 \times 10^{6}\right)$ were divided into three groups and injected into the mammary fat pad of mice. DHT treatment accelerated tumor growth significantly, while HSP27 knock-down decreased the tumor formation rate compared to the control group ( $83.3 \%$ vs. $100 \%)$ and inhibited tumor volume significantly (Fig. 6a and b).

In sections with hematoxylin-eosin (HE) staining, no obvious difference was found in the tissue structure and cell morphology of xenograft tumors generated from these three groups; however, necrosis could be found in the HSP27 knock-down group. Tumors generated from cells with DHT treatment expressed significantly higher Ki67 labeling, while tumors with HSP27 knock-down expressed lower Ki67 compared to the control group. Apoptosis of cancer cells was evaluated by TUNEL staining. Tumors generated from the DHT treatment group showed the smallest proportion of TUNELpositive cells, while the HSP27 knock-down group showed the largest proportion compared to the control group (Fig. 6c). In addition, no metastases were found in the lungs among all these three groups; however, interestingly, there were two mice that developed liver metastases in the DHT treatment group (Fig. 6d).

\section{Discussion}

Breast cancer is a heterogeneous tumor that can be reflected in different aspects, such as the classical histopathological features and the more modern molecular classification. MABC is defined as ER-negative, PR-negative, and AR-positive [12]; furthermore, with a poor prognosis by a series of clinical samples [3]. In this study, we further explored the mechanisms by which the AR regulates the malignancy of MABC. The results showed that HSP27 phosphorylation induced by androgen played a vital role in the process 


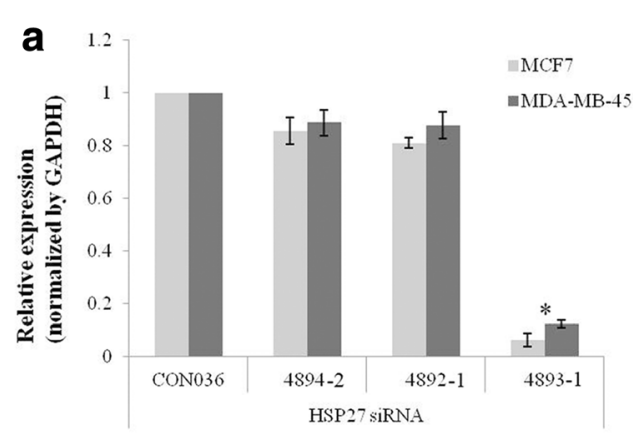

b

C

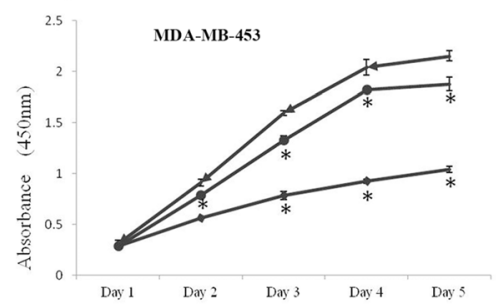

d
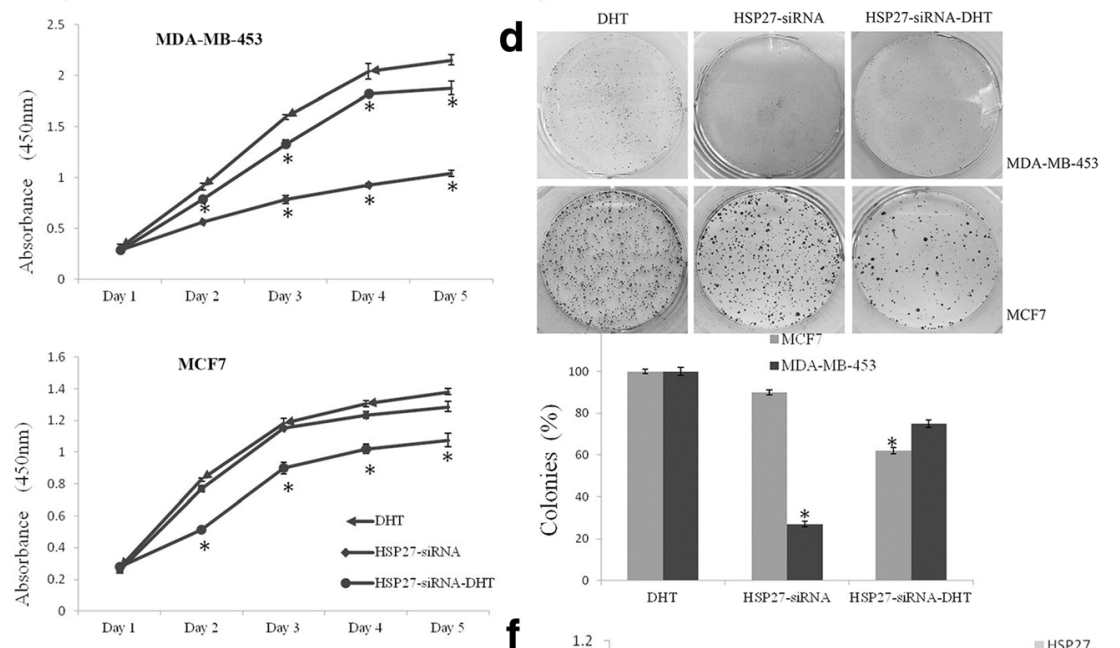

e
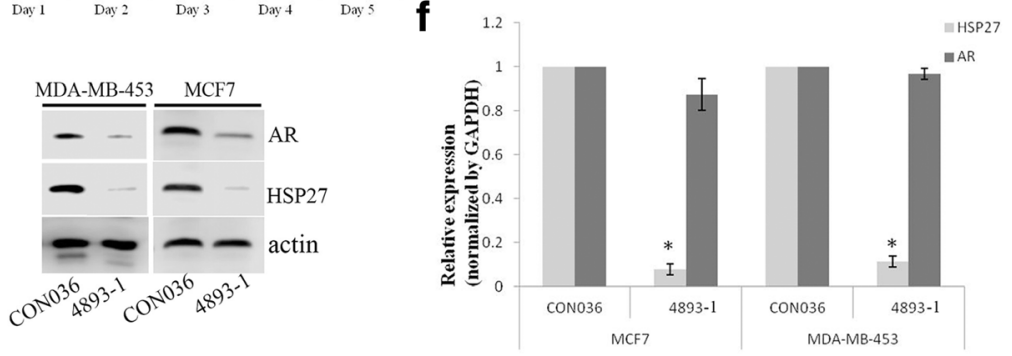

$\mathbf{g}$
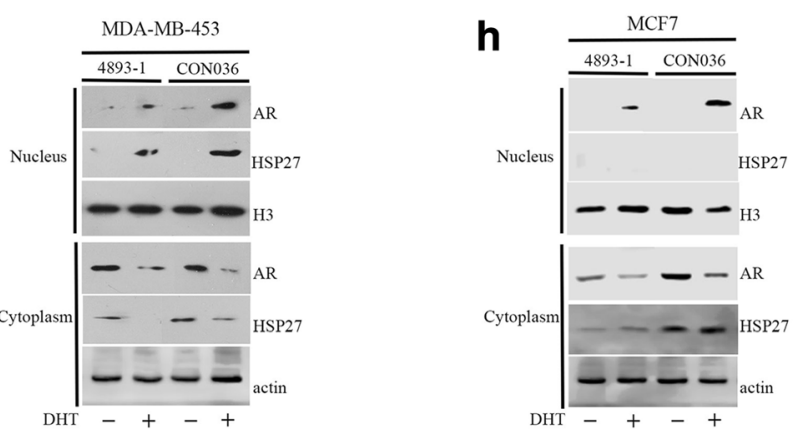

Fig. 4 HSP27 affected the expression and location of AR. Verification of the siRNA specific for HSP27 at three sites (4894-2, 4893-1, 4892-1) was measured by qPCR (a) and western blot (b). The proliferation ability of HSP27 knock-down cells treated with or without DHT was measured by CCK8 (c) and clonogenic (d) assays. The effect of HSP27 knock-down on AR expression in MDA-MB-453 and MCF7 cells was analyzed by western blot (e) and GPCR (f). Western blot analyzed the level of AR expression in the cytoplasm and nucleus after cells transfected with siRNA specific for HSP27 in MDA-MB-453 (g) and MCF7 (h) cells. ${ }^{*} P<0.05$

of AR translocation from the cytoplasm to the nucleus, which could affect the proliferation of tumor cells and tumorigenic capacity.
As a model of MABC, the proliferation of MDA-MB453 cells is regulated by androgen in an AR-dependent manner [13-15]. In MABC cells, HSP27 was highly 

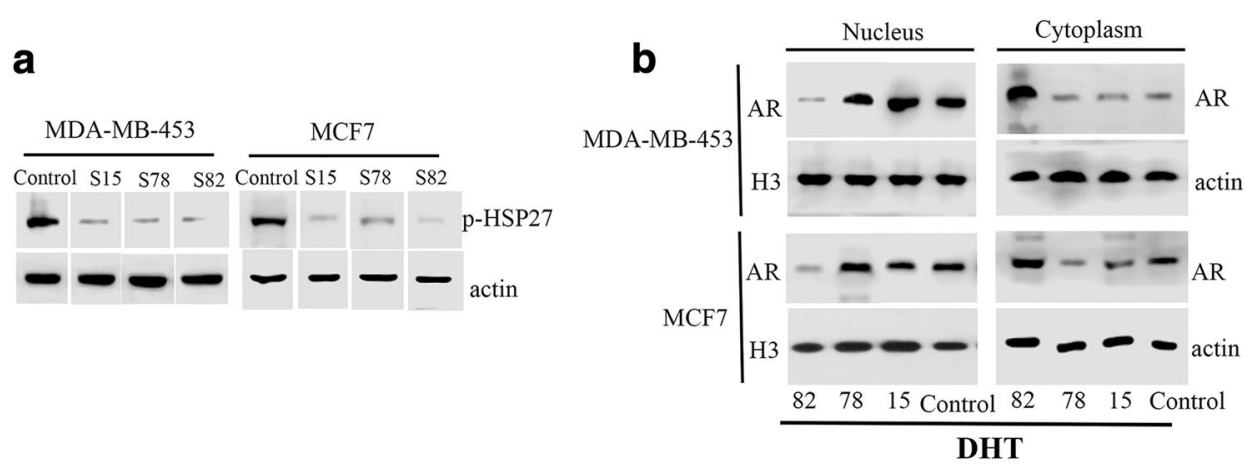

C

DAPI

AR

HSP27

Merge
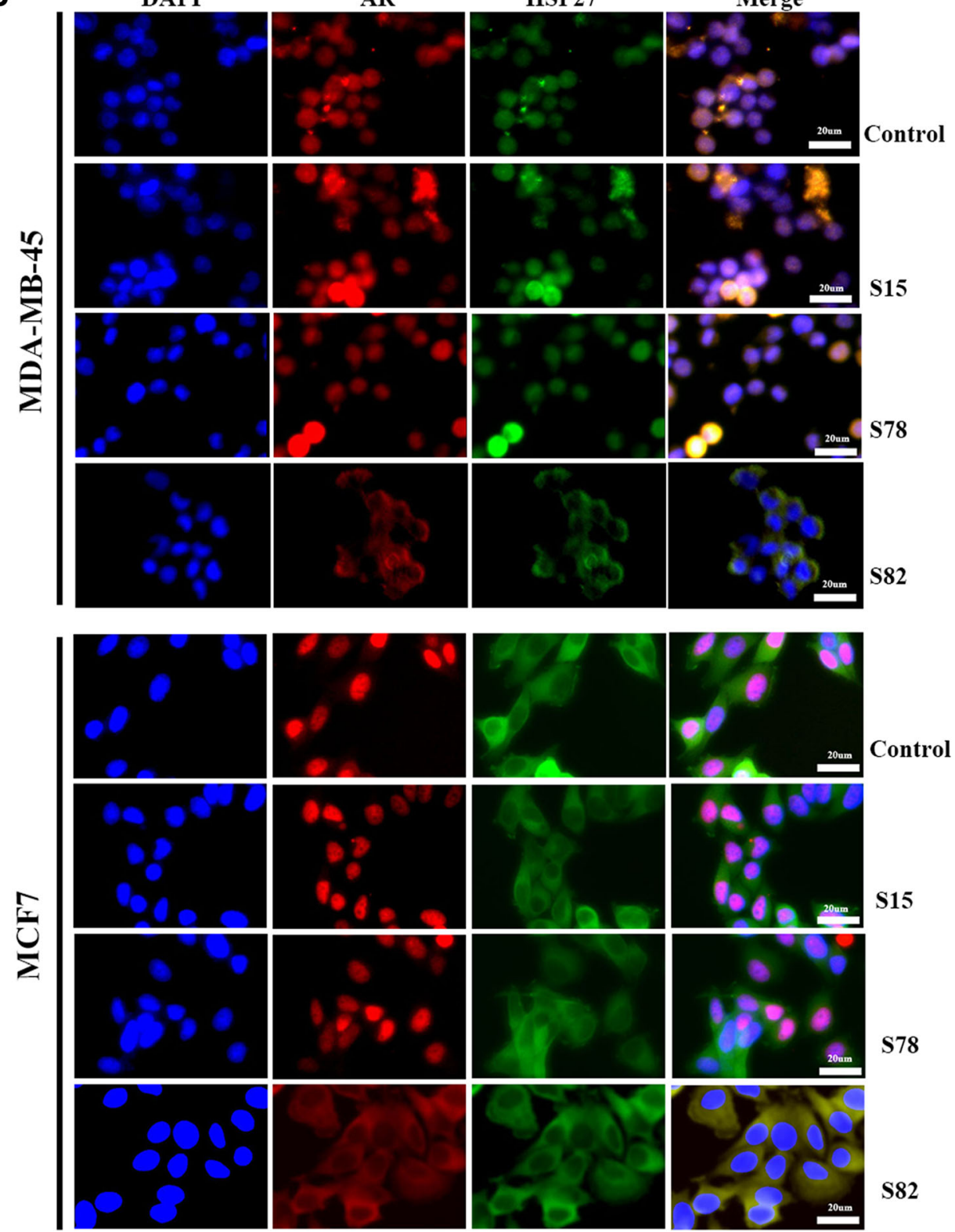

Fig. 5 The critical sites of HSP27 phosphorylation for AR cytoplasmic/nuclear translocation. Verification of deleting the residues of HSP27 phosphorylation sites (serine 15, 78, and 82) was measured by western blot (a). The expression and location of AR and HSP27 in the cytoplasm and nucleus after HSP27 phosphorylation sites deleted and treated with DHT were analyzed by western blot (b) and immunofluorescence (c) assays 


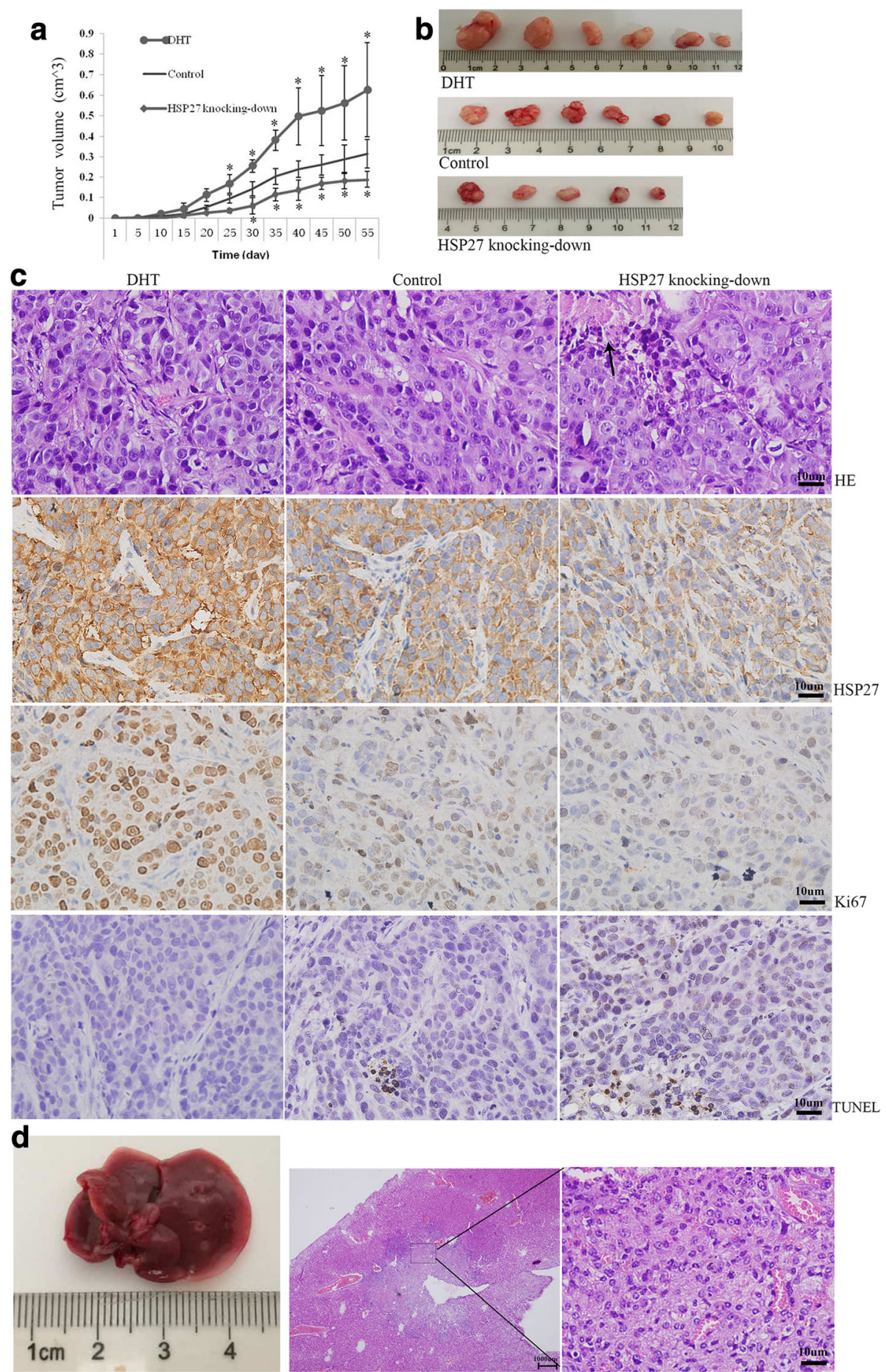

Fig. 6 HSP27 and DHT affected the tumor formation and metastasis in vivo using MDA-MB-453 cells. The effect of DHT and HSP27 knock-down on xenograft tumors growth and formation was described by tumor volume curve (a). Images of tumors removed from the mice (b). HE staining, immunohistochemistry staining for HSP27 and Ki67, and TUNEL staining were carried out on xenograft tumors (c). Representative images of liver with cancer cells invasion (d)

expressed and played a pivotal role in cell proliferation. In accordance with previous studies [2, 14, 16], we found that androgen could stimulate the proliferation of MDA-
MB-453 cells. In contrast to the promotion effect on MABC cells, androgen has a predominantly inhibitory proliferative effect on MCF7 cells [17], a model of 
luminal breast cancer [10]. However, the effects of androgen on MDA-MB-453 cells could be partly rescued by down-regulation of HSP27, suggesting that knockingdown HSP27 could reduce the androgen promotion effect on MDA-MB-453 cells. Additionally, HSP27 can decrease the toxic effects of oxidized proteins and reduce reactive oxygen species, which results in inhibiting cancer cell apoptosis [18-20]. These results suggest that androgen and HSP27 may interact with each other and co-regulate the proliferative ability of MABC cells.

HSP27 is a member of the HSPs family and has been stated to regulate the functions of the AR, such as AR cytoplasmic/nuclear translocation and AR transactivation $[6,21]$. Studies confirm that gain and loss of function of HSP27 are strongly related to the expression of the AR in myogenic cells and prostate cancer cells $[22,23]$. We also found that HSP27 knock-down significantly decreased the level of AR protein, but no difference could be found in the level of AR mRNA, which suggests that HSP27 might only regulate the protein translation of AR in MABC cells. However, Stope et al. [23] stated that the decrease in the AR protein level is paralleled with a down-regulation in $A R$ mRNA levels. This difference might be owing to the different cancer cells used for the research; however, the related mechanism should be further analyzed.

In malignant tumor cells, the expression of HSP27 is obviously high $[24,25]$. The main mechanism by which the AR and HSP27 regulate the malignant behavior of MABC may rely on the the androgen-triggered phosphorylation of HSP27 that accompanies the AR tanslocation from the cytoplasm to the nucleus where the AR interacts with androgen response elements to promote its genomic activity. HSP27 is directly phosphorylated at three serine residues via the $\mathrm{p} 38$ mitogen-activated protein kinase (MAPK) pathway, which affects its subcellular distribution [26-28]. The phosphorylation sites at serine 78 and 82 are identified as the predominant residues of HSP27, and serine 78 can be substituted by asparagine in some animals, but serine 82 is conserved throughout the animals [9]. In MABC cells, deleting residue serine 82 of the HSP27 phosphorylation sites induced a significant decrease in the expression levels of AR in the nucleus compared to the control group, which indicated that HSP27 phosphorylation at serine 82 might be the critical site for AR cytoplasmic and nuclear translocation.

Several studies have confirmed that the positive expression of AR is significantly associated with poor survival, and increased mortality in AR-positive triple negative breast cancer (TNBC) [3, 29-31]. HSP27 is reported to be associated with a decreased survival in breast cancer [32]. In accordance with clinical research, we found that DHT treatment increased xenograft tumor volume and distant metastasis, while HSP27 knock-down inhibited tumor growth greatly, which indicated that the AR and HSP27 might interact with each other and co-influence the development of MABC. Studies have stated that AR antagonists are able to induce breast cancer cell apoptosis and decrease tumor proliferation significantly in TNBC cell lines [33, 34]. In addition, the HSP second generation antisense oligonucleotide targeting HSP27 can increase drug efficacy in pancreatic and prostate cancer xenograft models [35-37]. Based on these results, although no valid endocrine therapy is suggested for MABC, the combination of AR antagonists and HSP27 inhibitors could be a potential therapeutic regimen. Of course, more xenograft models and clinical trials should be carried out to confirm this hypothesis.

\section{Conclusions}

In conclusion, this study confirmed that activation of AR and HSP27 by androgen could increase the proliferative ability of MABC cells and the growth of xenograft tumors. HSP27 phosphorylation on residue serine 82, induced by androgen, played a critical role in the process of AR translocation from the cytoplasm to the nucleus. The AR and HSP27 could form a protein complex, which was the main factor in AR regulating the malignancy behavior of tumor cells, and could present a new therapeutic regimen in clinical therapies of MABC.

\section{Additional file}

Additional file 1: The sequence of the deleted HSP27 phosphorylation sites. (DOCX $13 \mathrm{~kb}$ )

\section{Abbreviations}

AR: Androgen receptor; CCK8: Cell counter kit-8; Co-IP: Co-

immunoprecipitation; DCC-FBS: Dextran-coated charcoal-treated FBS; DHT: Dihydrotestosterone; ER: Estrogen receptor; HE: Hematoxylin-eosin; HSPS: Heat shock proteins; IF: Immunofluorescence; MABC: Molecular apocrine breast cancer; MAPK: Mitogen-activated protein kinase;

PR: Progesterone receptor; TNBC: Triple negative breast cancer; TUNEL: Terminal deoxynucleotidyl transferase dUTP nick end labeling

\section{Funding}

This work was financially supported by National Science Foundation of China (Grant number: 81172532).

Availability of data and materials

Please contact the corresponding author for all data requests.

Authors' contributions

YN and XZL conceived and designed the experiments. $X Z L$ and J J performed most of the experiments. CYF performed the statistical analysis. LC and GMX cultured the cells. FL, SLW and JJ performed some animal experiments. XZL, CYF and YN wrote the manuscript. All authors read and approved the final manuscript.

\section{Ethics approval and consent to participate}

For the in vivo tumor growth study, 18 mice were used. All studies were performed according to the American Association for the Accreditation of Laboratory Animal Care guidelines for humane treatment of animals and adhered to national and international standards. And it was approved by the 
Institution of Animal Use and Care Committee of Tianjin Medical University Cancer Institute and Hospital.

\section{Competing interests}

The authors declare that they have no competing interests.

\section{Publisher's Note}

Springer Nature remains neutral with regard to jurisdictional claims in published maps and institutional affiliations.

\section{Author details \\ ${ }^{1}$ Department of Breast Cancer Pathology and Research Laboratory, Tianjin Medical University Cancer Institute and Hospital, National Clinical Research Center of Cancer, Key Laboratory of Cancer Prevention and Therapy, Tianjin, Tianjin's Clinical Research Center for Cancer, Tianjin 300060, China. ${ }^{2}$ Key Laboratory of Breast Cancer Prevention and Therapy, Tianjin Medical University, Ministry of Education, West Huanhu Road, Ti Yuan Bei, Hexi District, Tianjin 300060, China. ${ }^{3}$ Department of Maternal and Child Health Hospital of Linyi, Qinghe-South Road, Luozhuang District, Linyi 276016, China.}

Received: 31 January 2018 Accepted: 17 April 2018 Published online: 27 April 2018

\section{References}

1. Farmer $\mathrm{P}$, Bonnefoi $\mathrm{H}$, Becette $\mathrm{V}$, Tubiana-Hulin M, Fumoleau $\mathrm{P}$, Larsimont $\mathrm{D}$, Macgrogan G, Bergh J, Cameron D, Goldstein D, Duss S, Nicoulaz AL, Brisken $\mathrm{C}$, et al. Identification of molecular apocrine breast tumours by microarray analysis. Oncogene. 2005;24:4660-71.

2. Doane AS, Danso M, Lal P, Donaton M, Zhang L, Hudis C, Gerald WL. An estrogen receptor-negative breast cancer subset characterized by a hormonally regulated transcriptional program and response to androgen. Oncogene. 2006:25:3994-4008

3. Liu X, Yang Y, Feng X, Shen H, Liu J, Liu X, Niu Y. Early versus late distant metastasis and adjuvant chemotherapy alone versus both radiotherapy and chemotherapy in molecular apocrine breast cancer. Oncotarget. 2016;7: 48905-17.

4. Robinson JL, Macarthur S, Ross-Innes CS, Tilley WD, Neal DE, Mills IG, Carroll JS. Androgen receptor driven transcription in molecular apocrine breast cancer is mediated by FoxA1. EMBO J. 2011;30:3019-27.

5. Proverbs-Singh T, Feldman JL, Morris MJ, Autio KA, Traina TA. Targeting the androgen receptor in prostate and breast cancer: several new agents in development. Endocr Relat Cancer. 2015;22:R87-R106.

6. Zoubeidi A, Zardan A, Beraldi E, Fazli L, Sowery R, Rennie P, Nelson C, Gleave M. Cooperative interactions between androgen receptor (AR) and heat-shock protein 27 facilitate AR transcriptional activity. Cancer Res. 2007; 67:10455-65.

7. Albany C, Hahn NM. Heat shock and other apoptosis-related proteins as therapeutic targets in prostate cancer. Asian J Androl. 2014;16:359-63.

8. Mymrikov EV, Seit-Nebi AS, Gusev NB. Large potentials of small heat shock proteins. Physiol Rev. 2011;91:1123-59.

9. Landry J, Lambert H, Zhou M, Lavoie JN, Hickey E, Weber LA, Anderson CW. Human HSP27 is phosphorylated at serines 78 and 82 by heat shock and mitogen-activated kinases that recognize the same amino acid motif as S6 kinase II. J Biol Chem. 1992;267:794-803.

10. Garbar C, Mascaux C, Giustiniani J, Merrouche Y, Bensussan A. Chemotherapy treatment induces an increase of autophagy in the luminal breast cancer cell MCF7, but not in the triple-negative MDA-MB231. Sci Rep. 2017;7:7201.

11. Naito S, von Eschenbach AC, Giavazzi R, Fidler IJ. Growth and metastasis of tumor cells isolated from a human renal cell carcinoma implanted into different organs of nude mice. Cancer Res. 1986:46:4109-15.

12. Lakis S, Kotoula V, Eleftheraki AG, Batistatou A, Bobos M, Koletsa T, Timotheadou E, Chrisafi S, Pentheroudakis G, Koutras A, Zagouri F, Linardou $H$, Fountzilas $G$. The androgen receptor as a surrogate marker for molecular apocrine breast cancer subtyping. Breast. 2014:23:234-43.

13. Ni M, Chen Y, Lim E, Wimberly H, Bailey ST, Imai Y, Rimm DL, Liu XS, Brown $M$. Targeting androgen receptor in estrogen receptor-negative breast cancer. Cancer Cell. 2011;20:119-31.
14. Naderi A, Hughes-Davies L. A functionally significant cross-talk between androgen receptor and ErbB2 pathways in estrogen receptor negative breast cancer. Neoplasia. 2008;10:542-8.

15. Birrell SN, Bentel JM, Hickey TE, Ricciardelli C, Weger MA, Horsfall DJ, Tilley WD. Androgens induce divergent proliferative responses in human breast cancer cell lines. J Steroid Biochem Mol Biol. 1995;52:459-67.

16. Cops EJ, Bianco-Miotto T, Moore NL, Clarke CL, Birrell SN, Butler LM, Tilley WD. Antiproliferative actions of the synthetic androgen, mibolerone, in breast cancer cells are mediated by both androgen and progesterone receptors. J Steroid Biochem Mol Biol. 2008;110:236-43.

17. Ortmann J, Prifti S, Bohlmann MK, Rehberger-Schneider S, Strowitzki T, Rabe T. Testosterone and 5 alpha-dihydrotestosterone inhibit in vitro growth of human breast cancer cell lines. Gynecol Endocrinol. 2002;16:113-20.

18. Rogalla T, Ehrnsperger M, Preville X, Kotlyarov A, Lutsch G, Ducasse C, Paul C. Wieske M, Arrigo AP, Buchner J, Gaestel M. Regulation of Hsp27 oligomerization, chaperone function, and protective activity against oxidative stress/tumor necrosis factor alpha by phosphorylation. J Biol Chem. 1999;274:18947-56.

19. Wyttenbach A, Sauvageot O, Carmichael J, Diaz-Latoud C, Arrigo AP, Rubinsztein DC. Heat shock protein 27 prevents cellular polyglutamine toxicity and suppresses the increase of reactive oxygen species caused by huntingtin. Hum Mol Genet. 2002;11:1137-51.

20. Shaw-Hallgren G, Chmielarska Masoumi K, Zarrizi R, Hellman U, Karlsson P, Helou K, Massoumi R. Association of nuclear-localized nemo-like kinase with heat-shock protein 27 inhibits apoptosis in human breast cancer cells. PLoS One. 2014;9:e96506.

21. Hassan S, Biswas MH, Zhang C, Du C, Balaji KC. Heat shock protein 27 mediates repression of androgen receptor function by protein kinase D1 in prostate cancer cells. Oncogene. 2009;28:4386-96.

22. Zhang Q, Lee HG, Kang SK, Baik M, Choi YJ. Heat-shock protein beta 1 regulates androgen-mediated bovine myogenesis. Biotechnol Lett. 2014;36:1225-31.

23. Stope MB, Schubert T, Staar D, Ronnau C, Streitborger A, Kroeger N, Kubisch C, Zimmermann U, Walther R, Burchardt M. Effect of the heat shock protein HSP27 on androgen receptor expression and function in prostate cancer cells. World J Urol. 2012;30:327-31.

24. Concannon CG, Gorman AM, Samali A. On the role of Hsp27 in regulating apoptosis. Apoptosis. 2003;8:61-70.

25. Haslbeck M, Franzmann T, Weinfurtner D, Buchner J. Some like it hot: the structure and function of small heat-shock proteins. Nat Struct Mol Biol. 2005;12:842-6.

26. Stokoe D, Engel K, Campbell DG, Cohen P, Gaestel M. Identification of MAPKAP kinase 2 as a major enzyme responsible for the phosphorylation of the small mammalian heat shock proteins. FEBS Lett. 1992;313:307-13.

27. Rouse J, Cohen P, Trigon S, Morange M, Alonso-Llamazares A, Zamanillo D, Hunt T, Nebreda AR. A novel kinase cascade triggered by stress and heat shock that stimulates MAPKAP kinase-2 and phosphorylation of the small heat shock proteins. Cell. 1994;78:1027-37.

28. Geum D, Son GH, Kim K. Phosphorylation-dependent cellular localization and thermoprotective role of heat shock protein 25 in hippocampal progenitor cells. J Biol Chem. 2002;277:19913-21.

29. Hu R, Dawood S, Holmes MD, Collins LC, Schnitt SJ, Cole K, Marotti JD, Hankinson SE, Colditz GA, Tamimi RM. Androgen receptor expression and breast cancer survival in postmenopausal women. Clin Cancer Res. 2011;17: 1867-74.

30. Lehmann BD, Bauer JA, Chen X, Sanders ME, Chakravarthy AB, Shyr Y, Pietenpol JA. Identification of human triple-negative breast cancer subtypes and preclinical models for selection of targeted therapies. J Clin Invest. 2011;121:2750-67

31. Choi JE, Kang SH, Lee SJ, Bae YK. Androgen receptor expression predicts decreased survival in early stage triple-negative breast cancer. Ann Surg Oncol. 2015;22:82-9.

32. Thanner F, Sutterlin MW, Kapp M, Rieger L, Morr AK, Kristen P, Dietl J, Gassel AM, Muller T. Heat shock protein 27 is associated with decreased survival in node-negative breast cancer patients. Anticancer Res. 2005:25:1649-53.

33. Zhu A, Li Y, Song W, Xu Y, Yang F, Zhang W, Yin Y, Guan X. Antiproliferative effect of androgen receptor inhibition in mesenchymal stem-like triplenegative breast cancer. Cell Physiol Biochem. 2016;38:1003-14.

34. Barton VN, D'Amato NC, Gordon MA, Lind HT, Spoelstra NS, Babbs BL, Heinz RE, Elias A, Jedlicka P, Jacobsen BM, Richer JK. Multiple molecular subtypes of triple-negative breast cancer critically rely on androgen receptor and respond to enzalutamide in vivo. Mol Cancer Ther. 2015;14:769-78. 
35. Rocchi P, Beraldi E, Ettinger S, Fazli L, Vessella RL, Nelson C, Gleave M. Increased Hsp27 after androgen ablation facilitates androgen-independent progression in prostate cancer via signal transducers and activators of transcription 3-mediated suppression of apoptosis. Cancer Res. 2005;65: 11083-93.

36. Rocchi P, So A, Kojima S, Signaevsky M, Beraldi E, Fazli L, Hurtado-Coll A, Yamanaka K, Gleave M. Heat shock protein 27 increases after androgen ablation and plays a cytoprotective role in hormone-refractory prostate cancer. Cancer Res. 2004;64:6595-602.

37. Baylot V, Andrieu C, Katsogiannou M, Taieb D, Garcia S, Giusiano S, Acunzo J, lovanna J, Gleave M, Garrido C, Rocchi P. OGX-427 inhibits tumor progression and enhances gemcitabine chemotherapy in pancreatic cancer. Cell Death Dis. 2011;2:e221.

Ready to submit your research? Choose BMC and benefit from:

- fast, convenient online submission

- thorough peer review by experienced researchers in your field

- rapid publication on acceptance

- support for research data, including large and complex data types

- gold Open Access which fosters wider collaboration and increased citations

- maximum visibility for your research: over $100 \mathrm{M}$ website views per year

At BMC, research is always in progress.

Learn more biomedcentral.com/submissions 